\title{
Crystalline Glucosamine Sulfate in the Treatment of Osteoarthritis: Evidence of Long-Term Cardiovascular Safety from Clinical Trials
} \author{
Albino Bonazzi and Lucio C. Rovati ${ }^{2}$ \\ ${ }^{1}$ Cardiology Department, Hospital Pulido Valente, CHLN, Lisboa, Portugal \\ ${ }^{2}$ Department of Clinical Pharmacology, Rottapharm|Madaus, Monza, Italy \\ ${ }^{3}$ Rheumatology Service, Hospital Militar Principal, Lisboa, Portugal
}

Roberto Palma dos Reis ${ }^{1}$, Giampaolo Giacovelli ${ }^{*}, 2$, Federica Girolami ${ }^{2}$, Rui André3,

\begin{abstract}
Background and Objective: Glucosamine is a safe and common treatment for osteoarthritis. Even so, literature data on the cardiovascular safety of glucosamine are limited. The objective of this paper is to investigate the long-term effects of crystalline glucosamine sulfate (CGS) on key measures of cardiovascular risk in patients with osteoarthritis.

Methods: We analyzed safety data from two long-term (6-month and 3-year, respectively) randomized controlled trials of CGS. Mean changes in blood pressure, lipids, and glucose were calculated for all patients randomized to CGS or placebo in either study and for subgroups with abnormally elevated baseline values. Shift tables were used to analyze transitions from normal to abnormal levels, or vice versa.

Results: This analysis on 428 osteoarthritis patients includes data from subjects who had, on average, high normal blood pressure or high cholesterol at baseline. There were no significant changes in mean blood pressure after 6 months on CGS (systolic: $-5 \pm 15 \mathrm{mmHg}$; diastolic: $-5 \pm 10 \mathrm{mmHg}$ ) or placebo (systolic: $-7 \pm 14 \mathrm{mmHg}$; diastolic: $-4 \pm 10 \mathrm{mmHg}$ ). Subgroup analysis did not show significant effects in subjects with hypertension. Likewise, blood lipids (total/LDL cholesterol) and blood glucose did not change over 3 years and 6 months of treatment, respectively, even in hypercholesterolemic or hyperglycemic subjects. The proportions of patients whose blood pressure or cholesterol levels shifted from normal to abnormal, or vice versa, were comparable in the CGS and placebo groups.
\end{abstract}

Conclusions: Long-term use of CGS did not affect blood pressure, lipids, or glucose in patients with osteoarthritis. These findings further support the cardiovascular safety of CGS.

Keywords: Glucosamine, osteoarthritis, safety, blood pressure, cholesterol, blood glucose.

\section{INTRODUCTION}

High blood pressure, high blood cholesterol, and diabetes are major risk factors for cardiovascular disease in many populations, especially in elderly subjects [1-4]. Clearly, this population has a high prevalence of multiple diseasesincluding osteoarthritis [5] - that must be managed at the same time and often require the use of different drugs. Authors from various affiliations have recently stressed the importance of evaluating the cardiovascular safety of drugs not prescribed for cardiovascular diseases. For instance nonsteroidal anti-inflammatory drugs (NSAIDs), which are commonly used by patients with osteoarthritis, can increase blood pressure. According to the European guidelines for the management of arterial hypertension, medications that can raise blood pressure should be monitored carefully [5-7].

Drugs for the treatment of osteoarthritis, the most common joint disease in older people, are classified as symptom-modifying agents and also as structure-modifying

\footnotetext{
*Address correspondence to this author at the Department of Clinical Pharmacology, Rottapharm|Madaus, via Valosa di Sopra 9, 20900 Monza, Italy; Tel: +39 0397390319; Fax: +39 0397390615 ; E-mail: giampaolo.giacovelli@rottapharm.com
}

agents if they can delay the progression of joint damage [5, 8]. Crystalline glucosamine sulfate (CGS; CAS number 216447-62-0) is a symptom-modifying drug with good evidence for favorable long-term effects on disease progression. CGS is a well-characterized substance containing glucosamine, sulfate, chloride, and sodium ions and showing tropism for articular cartilage [9]. It is approved as a prescription drug in Europe and elsewhere (Dona ${ }^{\circledR}$, Viartril-S ${ }^{\circledR}$ and other trade names of the Rottapharm|Madaus Group, Monza, Italy) and is marketed as a branded dietary supplement in the US. Extensively studied in randomized controlled clinical trials, CGS has a very good safety and tolerability profile, similar to that of placebo and significantly better than that of reference medications such as NSAIDs [10-15].

Among the methods whereby cardiovascular safety is assessed in clinical studies, measurements of blood pressure and blood lipid levels are regarded as key surrogate markers of cardiovascular risk [6]. These variables were not prospectively selected for statistical testing in clinical trials of CGS, mostly because of the lack of preclinical or clinical hints that could relate the use of glucosamine to hypertension or hypercholesterolemia. On the other hand, CGS composition includes a small amount, i.e. $384 \mathrm{mg}$, of sodium chloride. 
Its administration adds therefore to the dietary sodium intake and might contribute to increase blood pressure levels, especially in patients on a controlled sodium diet because of their hypertension/high normal blood pressure [7]. It was also speculated that glucosamine, which is an amino sugar, might lead to hyperglycemia and insulin resistance by overactivating the hexosamine pathway and impairing GLUT4 glucose transporter translocation to the plasma membrane [16]. Finally, alterations of glucose metabolism and dyslipidemia are frequently linked and, although there are no other clear mechanisms that may connect glucosamine administration with altered lipid metabolism, one report described 3 cases of increased cholesterol levels in patients receiving a glucosamine-based product [17]. Despite these concerns, and probably because glucosamine is traditionally regarded as safe, literature data on the cardiovascular safety of glucosamine-based products and in particular of the original prescription product consisting of CGS, are limited. Additional evidence-based references are warranted for a safe use of CGS in osteoarthritis patients at risk of cardiovascular diseases.

The objective of this report is therefore to provide more information on the cardiovascular safety of CGS through a retrospective analysis of blood pressure, blood lipid, and blood glucose data available from two high-quality randomized controlled clinical trials.

\section{MATERIALS AND METHODOLOGY}

The two clinical trials from which this analysis derives were conducted in accordance with the Declaration of Helsinki-the latest version available at the time each study was designed - and are briefly described below. They were approved by the Ethics Committee of each participating center, and written informed consent was obtained from each patient. Full details on these aspects, as well as on general methodology and inclusion/exclusion criteria, are given in the original publications by Herrero-Beaumont et al. (GUIDE study) and by Reginster et al. [10, 11].

\section{Design of the Original Studies}

Male and female outpatients fulfilling the clinical and radiological criteria of the American College of Rheumatology for the diagnosis of primary osteoarthritis were included in the studies [18]. Both trials were conducted according to a prospective, randomized, placebo-controlled (and reference-controlled in GUIDE), double-blind design. CGS was always used in its once daily formulation, i.e. sachets of powder for oral solution, containing $1500 \mathrm{mg}$ of glucosamine sulfate.

In the GUIDE study (ClinicalTrials.gov identifier: NCT00110474) [10], patients with knee osteoarthritis were randomized to receive CGS $1500 \mathrm{mg}$ once daily $(\mathrm{n}=109)$, acetaminophen (paracetamol) $3 \mathrm{~g}$ a day $(\mathrm{n}=109)$, or matching placebo $(n=107)$ for 6 months. In the long-term trial by Reginster and his group [11], patients with knee osteoarthritis were assigned to receive either CGS $1500 \mathrm{mg}$ once daily $(n=106)$ or matching placebo $(n=106)$ for 3 years.

\section{Methods Relevant to the Present Analysis}

Repeated blood pressure measurements were available from the GUIDE study. They were taken at randomization and then after 15 days and 1, 3, and 6 months of doubleblind treatment. Blood pressure was measured on the right arm, with the patient in a sitting position after 5 minutes of rest. Results are expressed in $\mathrm{mmHg}$.

Stored samples from the 3-year trial by Reginster and colleagues were used to test whether CGS had an effect on blood lipid levels. Blood samples were drawn at enrollment in the study and then at the end of each year of treatment. Total cholesterol, LDL cholesterol, HDL cholesterol, and triglyceride plasma levels were assayed by enzymatic techniques, as previously described by other authors [19]. All available samples from patients still in the study at each time point were assayed. However, because of the technical characteristics of the LDL cholesterol assay, slightly fewer samples were adequate for direct testing of this parameter. Missing LDL cholesterol values were therefore estimated by the Friedewald formula (i.e. LDL cholesterol $=$ total cholesterol - HDL cholesterol - triglycerides/5) [20]. Data are expressed as $\mathrm{mg} / \mathrm{dL}$ for all blood lipid variables.

Measurements of plasma glucose levels were available from the GUIDE study, in which fasting glucose concentrations were monitored at randomization and after 3 and 6 months of treatment in all patients still receiving the study medications. Plasma glucose was assayed by the glucose-oxidase GOD/PAP method (Roche Diagnostics, Mannheim, Germany), and data are expressed as mg/dL.

\section{Statistics}

For the purposes of this retrospective analysis we took into account the data available or obtainable from the double-blind period of recent randomized controlled trials of CGS. Because the present report is meant to evaluate the cardiovascular safety of CGS, all randomized patients were included in the Safety Population except for those assigned to acetaminophen (paracetamol) in the GUIDE trial. Unless otherwise stated, results are reported as mean and standard deviation (SD); $p$ values of 0.05 or less are considered statistically significant.

Regardless of the variable being tested, the primary endpoint was the mean absolute change (delta) from baseline at each evaluation time point. Statistical comparisons within and between groups were performed by one-way analysis of variance (ANOVA). No correction for multiple analyses was done in order not to miss any safety signal. For descriptive purposes, mean absolute values at baseline are reported for all available patients. Mean absolute values are also reported for patients having an actual measurement both at baseline and at the time of assessment. The primary analysis was conducted on all available patients from the Safety Population of each study.

Using the same method as described above, we also did a series of secondary analyses to assess the effects of CGS in the subgroups of patients who had abnormal blood pressure, blood lipid, or blood glucose levels at baseline. Widely accepted reference ranges were chosen to establish a cut-off between normal and abnormal values. Hypertensive patients were defined as those having systolic blood pressure $\geq 140$ $\mathrm{mmHg}$ and/or diastolic blood pressure $\geq 90 \mathrm{mmHg}$ [7]. Total cholesterol levels $\geq 200 \mathrm{mg} / \mathrm{dL}$ or LDL cholesterol levels $\geq 130 \mathrm{mg} / \mathrm{dL}$ were considered to be abnormally elevated lipid values [21, 22]. Glucose concentrations above the upper 
normal limit (UNL; $110 \mathrm{mg} / \mathrm{dL}$ ) were regarded as high blood glucose levels [23]. These analyses provide a clinically meaningful comparison in subgroups of patients who are already at risk and might therefore be more susceptible to negative changes.

Additionally, shift tables were used to evaluate how many patients, among those who had their blood pressure or blood lipid levels recorded both at baseline and at the end of treatment, shifted from normal to abnormal levels (or vice versa) following treatment with CGS or placebo. Comparison within each treatment group was done by the test of McNemar. This analysis provides an assessment of risk by taking into account natural fluctuations in measured values.

\section{RESULTS}

\section{Patient Populations}

325 and 212 osteoarthritis patients, respectively, were enrolled in the two clinical trials that are considered here. Of these, 109 were randomized to receive acetaminophen (paracetamol) and were therefore excluded from the present analysis, the Safety Population of which comprised 428 individuals overall. Baseline demographic and clinical characteristics were comparable between treatment groups in each study, as detailed in the original reports $[10,11]$.

\section{Blood Pressure}

To obtain meaningful information on the cardiovascular safety of CGS, we first analyzed blood pressure changes in patients who participated in the GUIDE study. On average, this population had high normal blood pressure, with no differences between treatment groups at baseline. There were no significant changes in blood pressure during the 6-month study period. However, mean systolic and diastolic values tended to decrease over time in both the CGS and the placebo groups, as evidenced by the consistent negative sign of the change from baseline at each evaluation time point (Table 1).
The shift table shows a similar pattern for CGS and placebo. Indeed, the proportion of patients whose blood pressure values shifted from normal at baseline to hypertensive at the end of treatment (about 7\% in each group) was outbalanced by a higher proportion of patients showing an opposite trend (about $20 \%$ in each arm). The McNemar test revealed that the subjects who improved were significantly more than those who worsened (Table 2).

Next, we did a subgroup analysis to find out whether CGS had the potential to further impair blood pressure in individuals with arterial hypertension. At baseline, 104 hypertensive subjects were identified in the placebo and CGS groups of GUIDE ( $n=51$ and $n=53$, respectively). Paired baseline and 6-month measurements were available for 79 such individuals (placebo, $n=41$; CGS, $n=38$ ). Mean baseline values for systolic and diastolic blood pressure were $149 \pm 11$ and $84 \pm 10 \mathrm{mmHg}$ in the placebo group, and $148 \pm 10$ and $87 \pm 10 \mathrm{mmHg}$ in the CGS arm. In agreement with the results observed in the general study population, systolic and diastolic blood pressure in hypertensive patients did not change significantly but tended to decrease. Mean changes were $-11 \pm 13$ and $-6 \pm 9 \mathrm{mmHg}$ respectively in subjects on placebo, and $-10 \pm 18$ and $-8 \pm 10 \mathrm{mmHg}$ in those on CGS.

\section{Blood Lipids}

Secondly, to assess whether long-term exposure to CGS could adversely affect cholesterol and triglyceride levels, we examined blood lipid changes in subjects who participated in the 3-year study by Reginster and his group. At baseline, mean total and LDL cholesterol concentrations were above the normal range in this population, with no differences between treatment groups. No significant changes in total or LDL cholesterol were observed during the long-term treatment period with placebo or CGS (Table 3).

The proportion of patients whose total cholesterol levels shifted from normal at baseline to abnormal (high) after 3 years was comparable in the placebo and CGS groups (around 10\%). Additionally, it was well balanced by a similar proportion of patients with an opposite trend $(11.1 \%$

Table 1. Systolic and Diastolic Blood Pressure (Expressed in mmHg) During the GUIDE Study

\begin{tabular}{|l|l|c|c|c|c|}
\hline \multirow{2}{*}{ Evaluation Time } & \multirow{2}{*}{ Study Treatment } & \multicolumn{2}{|c|}{ Baseline Values* } & \multicolumn{2}{|c|}{ Delta at Each Time Point } \\
\cline { 2 - 6 } & & Systolic & Diastolic & \multicolumn{2}{|c|}{ Systolic } \\
\hline \hline \multirow{3}{*}{ Baseline } & Placebo (n=107) & $136(16)$ & $78(11)$ & - & - \\
\cline { 2 - 6 } & CGS (n=109) & $134(15)$ & $79(11)$ & - & $-1(9)$ \\
\hline \multirow{3}{*}{ Day 15 } & Placebo (n=104) & $137(16)$ & $78(11)$ & $-2(12)$ & $-1(8)$ \\
\cline { 2 - 6 } & CGS (n=105) & $134(15)$ & $79(11)$ & $-1(13)$ & $-2(10)$ \\
\hline \multirow{3}{*}{ Month 3 } & Placebo (n=97) & $136(16)$ & $78(11)$ & $-2(15)$ & $-1(8)$ \\
\cline { 2 - 6 } & CGS (n=97) & $134(16)$ & $78(12)$ & $-3(13)$ & $-1(11)$ \\
\hline \multirow{2}{*}{ Month 6 } & Placebo (n=92) & $134(16)$ & $78(11)$ & $-7(14)$ \\
\cline { 2 - 6 } & CGS (n=93) & $137(16)$ & $78(11)$ & $-4(10)$ \\
\hline
\end{tabular}

Values are expressed as mean (SD); $p=$ not significant in all comparisons.

*In this column, the average of absolute values at baseline for the patients still in study at each time point is given.

$\mathrm{CGS}=$ crystalline glucosamine sulfate. 
Table 2. Patients Shifting from Normal to Abnormal (Hypertensive) Blood Pressure Values, and Vice Versa, from Baseline to the End of Treatment (Month 6)

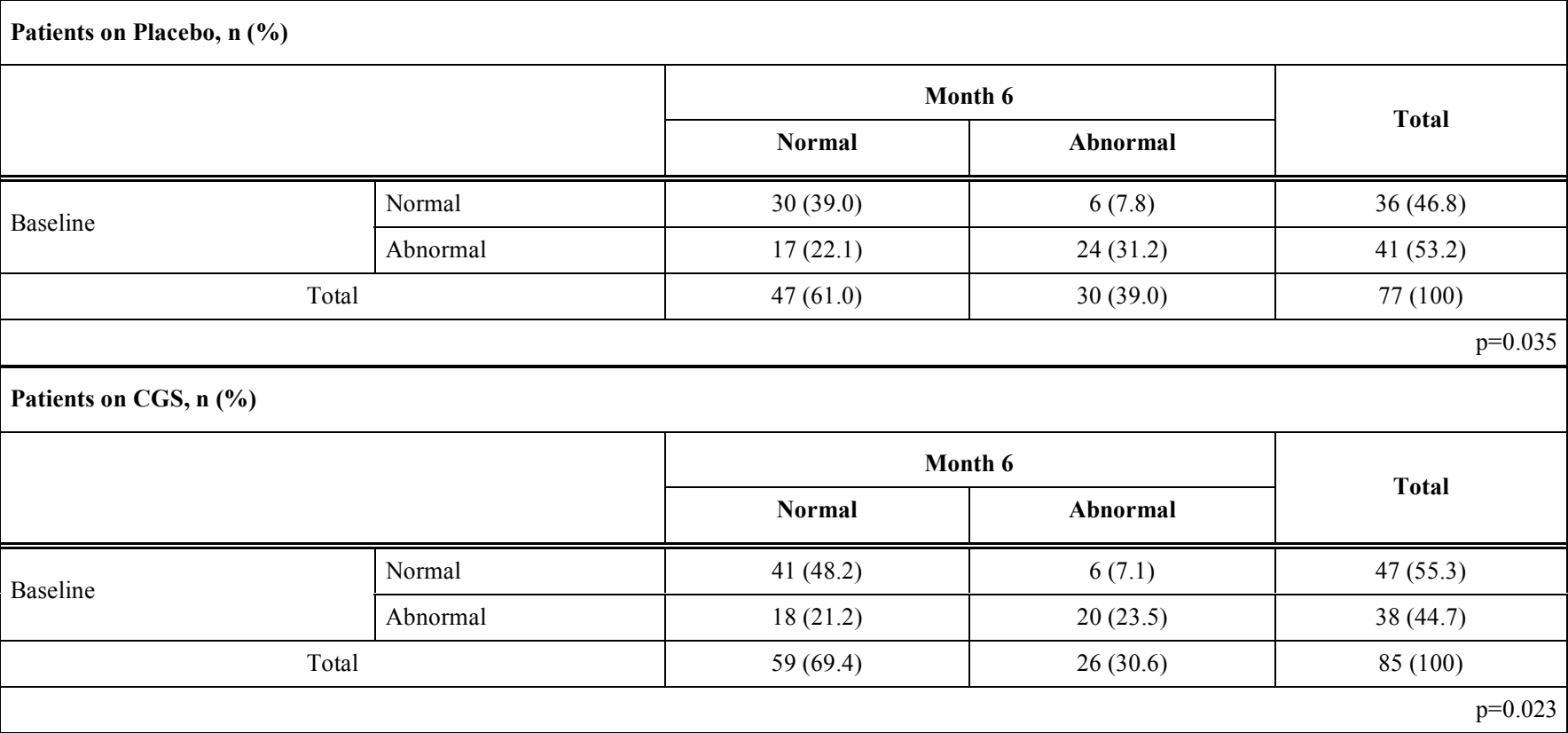

This analysis takes into account patients for whom both values (baseline and 6-month) were available.

Statistical analysis was done by McNemar test.

CGS=crystalline glucosamine sulfate.

Table 3. Total and LDL Cholesterol Levels (Expressed as mg/dL) During the Long-Term Trial by Reginster et al.

\begin{tabular}{|c|c|c|c|c|}
\hline Evaluation Time & Study Treatment & Baseline Values* & Values at Each Time Point & Delta at Each Time Point \\
\hline \multicolumn{5}{|c|}{ Total Cholesterol } \\
\hline \multirow{2}{*}{ Baseline } & Placebo $(n=70)$ & $224(40)$ & - & - \\
\hline & CGS $(n=91)$ & $219(32)$ & - & - \\
\hline \multirow{2}{*}{ Year 1} & Placebo $(\mathrm{n}=56)$ & $226(40)$ & $221(48)$ & $-5(41)$ \\
\hline & CGS $(n=77)$ & $219(33)$ & $223(33)$ & $4(33)$ \\
\hline \multirow{2}{*}{ Year 2} & Placebo $(\mathrm{n}=47)$ & $220(37)$ & $225(37)$ & $4(37)$ \\
\hline & CGS $(n=66)$ & $219(32)$ & $222(35)$ & $3(31)$ \\
\hline \multirow{2}{*}{ Year 3} & Placebo $(n=36)$ & $220(38)$ & $221(38)$ & $1(35)$ \\
\hline & CGS $(n=62)$ & $216(30)$ & $216(35)$ & $0(37)$ \\
\hline \multicolumn{5}{|c|}{ LDL Cholesterol } \\
\hline \multirow{2}{*}{ Baseline } & Placebo $(n=70)$ & $154(41)$ & - & - \\
\hline & CGS (n=92) & $161(34)$ & - & - \\
\hline \multirow{2}{*}{ Year 1} & Placebo $(n=56)$ & $155(40)$ & $153(39)$ & $-2(33)$ \\
\hline & CGS $(n=78)$ & $161(34)$ & $159(36)$ & $-3(32)$ \\
\hline \multirow{2}{*}{ Year 2} & Placebo $(n=47)$ & $147(36)$ & $148(36)$ & $1(32)$ \\
\hline & CGS $(n=66)$ & $161(33)$ & $161(35)$ & $-1(24)$ \\
\hline \multirow{2}{*}{ Year 3} & Placebo $(n=36)$ & $147(38)$ & $140(34)$ & $-7(31)$ \\
\hline & CGS $(n=63)$ & 157 (29) & $153(34)$ & $-5(31)$ \\
\hline
\end{tabular}

Values are given as mean (SD); $\mathrm{p}=$ not significant in all comparisons.

*In this column, the average of absolute values at baseline for the patients still in study at each yearly clinic visit is given.

CGS $=$ crystalline glucosamine sulfate.

and $14.5 \%$ after placebo and CGS, respectively). Likewise, no differences were found in the distribution of normal and high LDL cholesterol levels between the beginning and the end of treatment (Table 4). 
Table 4. Patients Shifting from Normal to Abnormal (Elevated) Cholesterol Levels, and Vice Versa, from Baseline to the End of Treatment (Year 3)

\begin{tabular}{|c|c|c|c|c|c|}
\hline \multicolumn{6}{|c|}{ Total Cholesterol } \\
\hline \multicolumn{6}{|c|}{ Patients on Placebo, n (\%) } \\
\hline & & & & \multirow{2}{*}{\multicolumn{2}{|c|}{ Total }} \\
\hline & & Normal & Abnormal & & \\
\hline \multirow{2}{*}{ Baseline } & Normal & $6(16.7)$ & $3(8.3)$ & $9(25.0)$ & \\
\hline & Abnormal & $4(11.1)$ & $23(63.9)$ & $27(75.0)$ & \\
\hline \multicolumn{2}{|c|}{ Total } & $10(27.8)$ & $26(72.2)$ & \multicolumn{2}{|l|}{$36(100)$} \\
\hline & & & & & $\mathrm{p}=\mathrm{NS}$ \\
\hline \multicolumn{6}{|c|}{ Patients on CGS, n (\%) } \\
\hline & & & & \multirow{2}{*}{\multicolumn{2}{|c|}{ Total }} \\
\hline & & Normal & Abnormal & & \\
\hline \multirow{2}{*}{ Baseline } & Normal & $12(19.4)$ & $7(11.3)$ & \multicolumn{2}{|l|}{$19(30.6)$} \\
\hline & Abnormal & $9(14.5)$ & $34(54.8)$ & \multicolumn{2}{|l|}{$43(69.4)$} \\
\hline \multicolumn{2}{|c|}{ Total } & $21(33.9)$ & $41(66.1)$ & \multicolumn{2}{|l|}{$62(100)$} \\
\hline & & & & & $\mathrm{p}=\mathrm{NS}$ \\
\hline \multicolumn{6}{|c|}{ LDL Cholesterol } \\
\hline \multicolumn{6}{|c|}{ Patients on placebo, n (\%) } \\
\hline & & & & \multirow{2}{*}{\multicolumn{2}{|c|}{ Total }} \\
\hline & & Normal & Abnormal & & \\
\hline \multirow{2}{*}{ Baseline } & Normal & $8(22.2)$ & $4(11.1)$ & \multicolumn{2}{|l|}{$12(33.3)$} \\
\hline & Abnormal & $5(13.9)$ & $19(52.8)$ & \multicolumn{2}{|l|}{$24(66.7)$} \\
\hline \multicolumn{2}{|c|}{ Total } & $13(36.1)$ & $23(63.9)$ & \multicolumn{2}{|l|}{$36(100)$} \\
\hline & & & & & $\mathrm{p}=\mathrm{NS}$ \\
\hline \multicolumn{6}{|c|}{ Patients on CGS, n (\%) } \\
\hline & & \multicolumn{2}{|c|}{ Year 3} & \multirow{2}{*}{\multicolumn{2}{|c|}{ Total }} \\
\hline & & Normal & Abnormal & & \\
\hline \multirow{2}{*}{ Baseline } & Normal & $7(11.1)$ & $5(7.9)$ & \multicolumn{2}{|l|}{$12(19.0)$} \\
\hline & Abnormal & $9(14.3)$ & $42(66.7)$ & $51(81.0)$ & \\
\hline \multicolumn{2}{|c|}{ Total } & $16(25.4)$ & $47(74.6)$ & \multicolumn{2}{|l|}{$63(100)$} \\
\hline
\end{tabular}

This analysis takes into account patients for whom both values (baseline and 3-year) were available.

NS=not significant by McNemar test.

CGS=crystalline glucosamine sulfate

Subgroup analysis of individuals with elevated cholesterol at baseline substantiated the results observed in the general study population. In this trial there were 114 patients in whom total cholesterol levels were elevated before treatment began (49 and 65 in the placebo and CGS groups, respectively). Seventy paired baseline and 3-year measurements of total cholesterol were available for analysis (placebo, $n=27$; CGS, $n=43$ ). Mean baseline values were $237 \pm 27$ and $231 \pm 21 \mathrm{mg} / \mathrm{dL}$ in subjects assigned to placebo and CGS, respectively. After 3 years there were no significant changes in total cholesterol, which tended to decrease in both arms of the study. The average difference was $-8 \pm 34 \mathrm{mg} / \mathrm{dL}$ in the placebo subgroup and $-9 \pm 28 \mathrm{mg} / \mathrm{dL}$ in the CGS subgroup. Similar results were seen in patients who had elevated LDL cholesterol at baseline (126 in total and 75 for whom baseline and 3-year measurements were available), with no significant changes between or within groups. Mean differences between final and baseline LDL cholesterol levels were $-18 \pm 30$ and $-9 \pm 29 \mathrm{mg} / \mathrm{dL}$ in the placebo and CGS arms, respectively. 
On average, patients participating in the Reginster's trial had borderline low HDL cholesterol, which was significantly lower in the CGS arm than in the placebo group at baseline. Fig. (1) shows that HDL cholesterol tended to increase in both groups, although the change was greater in patients on CGS than in those on placebo after 1 year $(4 \pm 7$ vs $1 \pm 9$ $\mathrm{mg} / \mathrm{dL} ; \mathrm{p}<0.05)$ and greater in the placebo group than in the CGS arm after 3 years $(6 \pm 7$ vs $2 \pm 9 \mathrm{mg} / \mathrm{dL} ; \mathrm{p}<0.05)$. Mean triglyceride levels were in the normal range at baseline and did not change throughout the 3 years of double-blind treatment (Fig. 1).

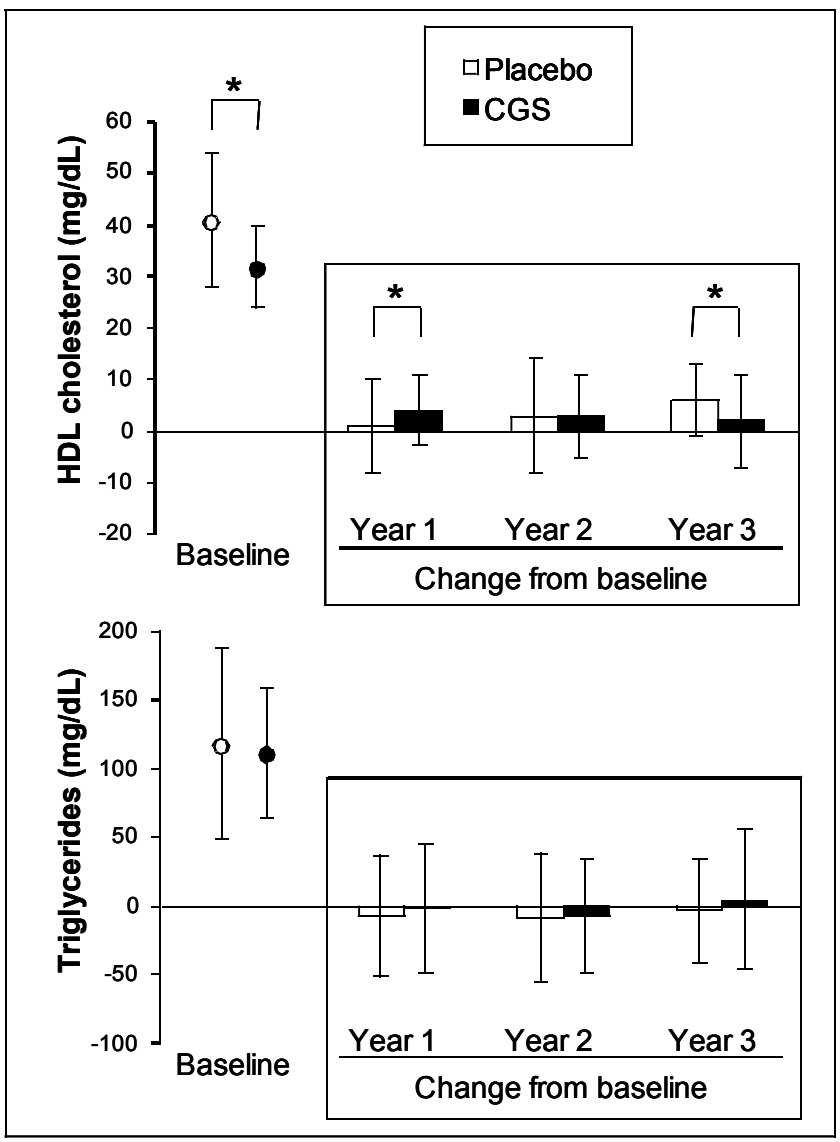

Fig. (1). Baseline levels and changes from baseline for HDL cholesterol and triglycerides. Stored blood samples from the 3-year trial by Reginster and colleagues were used to test whether GCS had an effect on blood lipids. Data are presented as mean \pm SD of all available measurements. ${ }^{*} \mathrm{p}<0.05$ between groups.

\section{Blood Glucose}

Finally, to further confirm that therapeutic doses of CGS do not impair glucose metabolism, we analyzed fasting glucose changes in subjects who were randomized to receive placebo or CGS in the GUIDE study. On average their plasma glucose was within the normal range at baseline. Table 5 shows that mean plasma glucose levels were virtually unaltered between screening and the 3- or 6-month assessment in both study groups.

Hyperglycemic subjects, i.e. those with plasma glucose levels above UNL at baseline, were examined in a separate analysis. CGS did not worsen glucose metabolism in this subgroup of patients. Rather, their mean glucose levels tended to decrease over time (Table 6). As expected, plasma glucose levels were unaffected in the subgroup receiving placebo.

\section{DISCUSSION}

The evidence presented here shows that long-term use of crystalline glucosamine sulfate (CGS) in randomized controlled trials did not adversely affect blood pressure, blood lipids, or blood glucose in patients with osteoarthritis. Because changes in these variables are predictors of cardiovascular risk [6], the present study provides new information supporting the excellent safety profile of CGS.

Monitoring the cardiovascular safety of medicines, even if they are not intended to treat cardiovascular diseases, is a priority going well beyond drug development and approval. NSAIDs are a good example of drugs that, while approved and widely prescribed for use in osteoarthritis, have been found to increase the risk of cardiovascular disease [6, 24]. Notably, the European guidelines for the management of hypertension include NSAIDs in a list of agents that can raise blood pressure. The same guidelines recommend that lifestyle changes, including reduction of salt intake, be instituted in hypertensive patients and in subjects who have high normal blood pressure [7]. Any drug product containing sodium salts, therefore, should be evaluated for its potential to increase blood pressure.

In its once daily formulation, CGS contains $1500 \mathrm{mg}$ of glucosamine sulfate and a small amount-384 $\mathrm{mg}$ - of sodium chloride. This fact must be taken into account by patients on a controlled sodium diet but, to date, the effects of CGS (or of glucosamine in general) on blood pressure have not been assessed systematically. Little information is available from the literature. In one cross-sectional study, long-term users of multiple dietary supplements, including glucosamine, were less likely to have high blood pressure than non-users [25]. An experimental study reports that systolic blood pressure was lower in normal or hypertensive rats treated with glucosamine for 9 weeks than in matching control animals [26]. Our present analysis shows that 6 months of treatment with CGS or placebo did not increase systolic or diastolic blood pressure. Rather, their mean values tended to decrease both in the general population of the GUIDE study and in the subgroup of patients who were hypertensive at baseline. Although the proportion of hypertensive subjects was significantly lower at month 6 than at baseline, we do not consider this finding to be clinically relevant. Remarkably, patients participating in the trial had high normal blood pressure on average, thus falling (as a group) within the classes of subjects for whom salt intake should be reduced [7]. The study population was therefore appropriate for the assessment of potential risks induced by treatment with CGS.

Likewise, the 3-year Reginster's trial was considered adequate for the evaluation of blood lipid changes after longterm exposure to CGS. There are no mechanism-based literature data supporting a link between glucosamine use and hypercholesterolemia. However, we found an article reporting 3 cases of increased cholesterol levels in patients taking glucosamine, but the author neither considered alternative explanations nor provided scientific grounds for a causal relationship between the drug and the adverse event [17]. Actually, because of the concern raised in that 
Table 5. Fasting Plasma Glucose (Expressed as mg/dL) During the GUIDE Study

\begin{tabular}{|l|l|c|c|c|}
\hline \multicolumn{1}{|c|}{ Evaluation Time } & \multicolumn{1}{|c|}{ Study Treatment } & Baseline Values* & Values at Each Time Point & Delta at Each Time Point \\
\hline \hline \multirow{2}{*}{ Baseline } & Placebo (n=100) & $99(14)$ & - & - \\
\cline { 2 - 5 } & CGS (n=98) & $98(15)$ & - & - \\
\hline \multirow{2}{*}{ Month 3 } & Placebo (n=82) & $99(15)$ & $101(16)$ & $2(9)$ \\
\cline { 2 - 5 } & CGS (n=77) & $97(15)$ & $97(15)$ & $0(9)$ \\
\hline \multirow{2}{*}{ Month 6 } & Placebo (n=74) & $99(15)$ & $102(16)$ & $3(10)$ \\
\cline { 2 - 5 } & CGS (n=76) & $98(15)$ & $98(15)$ & $0(9)$ \\
\hline
\end{tabular}

Values are expressed as mean (SD); $p=$ not significant in all comparisons.

*In this column, the average of absolute values at baseline for the patients still in study at each time point is given.

CGS $=$ crystalline glucosamine sulfate.

Table 6. Fasting Plasma Glucose (Expressed as mg/dL) During the GUIDE Study in Patients with Baseline Levels Above the Upper Normal Limit (UNL; $110 \mathrm{mg} / \mathrm{dL}$ )

\begin{tabular}{|l|l|c|c|}
\hline \multirow{2}{*}{ Evaluation Time } & \multicolumn{1}{|c|}{ Study Treatment (n=tot pts; $\mathbf{n}=\mathbf{p t s}>\mathbf{U N L})$} & Values at Each Time Point & Delta at Each Time Point \\
\hline \hline \multirow{2}{*}{ Baseline } & Placebo $(\mathrm{n}=15 ; \mathrm{n}=15)$ & $124(12)$ & - \\
\cline { 2 - 4 } & CGS $(\mathrm{n}=12 ; \mathrm{n}=12)$ & $129(17)$ & - \\
\hline \multirow{3}{*}{ Month 3 } & Placebo $(\mathrm{n}=14 ; \mathrm{n}=12)$ & $125(14)$ & $2(9)$ \\
\cline { 2 - 5 } & CGS $(\mathrm{n}=10 ; \mathrm{n}=7)$ & $122(22)$ & $-4(10)$ \\
\hline \multirow{2}{*}{ Month 6 } & Placebo $(\mathrm{n}=14 ; \mathrm{n}=12)$ & $125(15)$ & $1(10)$ \\
\cline { 2 - 5 } & CGS $(\mathrm{n}=10 ; \mathrm{n}=7)$ & $120(20)$ & $-6(8)$ \\
\hline
\end{tabular}

Values are expressed as mean (SD); $\mathrm{p}=$ not significant in all comparisons.

$\mathrm{n}=$ tot pts: number of patients with an abnormal glucose value at baseline and, of these, number of patients whose glucose levels were available after 3 and 6 months.

$\mathrm{n}=\mathrm{pts}>\mathrm{UNL}$ : number of patients with a glucose level above UNL at each time point.

publication, other authors did a post-marketing 3-month, randomized, double-blind, placebo-controlled study to investigate whether glucosamine sulfate could worsen lipid metabolism [27]. They concluded that glucosamine sulfate does not affect fasting plasma levels of cholesterol (total, LDL, and HDL) and triglycerides. Our findings not only support such conclusions but also extend them to a much longer exposure period and to hypercholesterolemic patients.

With regard to "good" cholesterol, although in vitro studies have shown that glucosamine stabilizes the mRNA for the main protein constituent of HDL (i.e. apoAI) [28], CGS did not increase HDL cholesterol in subjects with osteoarthritis. On average, HDL values were borderline low at baseline and did not change significantly over time. Again our results are in line with those obtained by other investigators, who demonstrated that glucosamine does not have significant effects on lipid profile and glycemic control in people with low HDL cholesterol and diabetes [29].

Our data on glucose homeostasis originate from the GUIDE trial, in which mean glucose levels were unchanged in all groups during the 6-month treatment. We recognize that single measures of fasting plasma glucose are far from being accurate in predicting the risk of diabetes or cardiovascular disease [23]. Yet, the effects of glucosamine on glucose metabolism have been studied extensively over the past two decades.

The concern that exogenous glucosamine might alter glycemic control by over-activating the hexosamine pathway was raised following experimental studies in which largely supra-pharmacological amounts of the compound were given intravenously to rats. Under these conditions, glucosamine impaired insulin secretion and induced insulin resistance by decreasing glucose uptake $[16,30]$. Insulin resistance often occurs in the hyperglycemic state, is a risk factor for diabetes, and is best assessed by euglycemic hyperinsulinemic clamp. At least three clinical trials used this reference method to examine the effects of oral, intraarterial, or intravenous glucosamine [31-33]. At variance with experimental models, these studies do not support a role for the hexosamine pathway or glucosamine (even at suprapharmacological doses) in the regulation of insulin sensitivity in humans. A comparison of in vitro data with human pharmacokinetic parameters confirms the above findings. In cultured L6 muscle cells, glucosamine decreases glucose uptake at concentrations higher than $5 \times 10^{-3} \mathrm{M}$ [34]. When CGS is administered at therapeutic doses, it provides a mean maximum plasma concentration $\left(\mathrm{C}_{\max }\right)$ of about $9 \times 10^{-6}$ $\mathrm{M}[35]$.

The majority of clinical studies to date, some of which included people with type 1 or 2 diabetes, have shown that therapeutic doses of glucosamine do not alter glucose metabolism in humans [29, 31, 32, 36, 37]. This position has been endorsed by recent reviews on the topic [38-40]. But we do not want to ignore the few studies hypothesizing that glucosamine may affect glucose metabolism, at least in subjects with untreated diabetes or glucose intolerance [41, 42]. Our current findings are reassuring on this point, 
because blood glucose did not worsen in the subgroup of hyperglycemic patients receiving CGS for 6 months. However, longitudinal data on diabetic patients are limited, and monitoring of blood sugar levels is recommended as a precaution when CGS is administered in such individuals.

Taken together, our data show that CGS was as safe as placebo in randomized controlled trials and did not change key markers of cardiovascular health. The analysis presented here, like any study, has both strengths and limitations. Indeed, this is the first report providing detailed statistics on blood pressure levels in osteoarthritis patients, either hypertensive or non-hypertensive, during treatment with CGS. The main limitation lies in the nature of a retrospective analysis. The original studies were designed to show clinical efficacy in osteoarthritis - and they did it - but may be undersized to detect small changes in the safety variables monitored. Still, our findings are consistent with those gathered in the last 15 years of post-marketing experience and surveillance. In the period between 1995 and 2010, a roughly estimated 29 million patients were treated with CGS, based on an average 3-month treatment period. By combining figures on patient exposure with the Company's global Pharmacovigilance database, containing spontaneous Individual Case Safety Reports (ICSRs) collected worldwide since 1995, an incidence below $1 / 1,000,000$ is found for the terms "increased blood pressure" $(n=28)$, "increased cholesterol/lipid levels" (n=03), and "increased glucose" $(\mathrm{n}=18)$. These figures are obtained including medicallyrelated terms and using a conservative approach (i.e. all ICSRs are taken into consideration regardless of the causality relationship with CGS, and even non-medically validated reports are included in the calculation).

\section{CONCLUSION}

This report provides new evidence supporting the cardiovascular safety of CGS in the long-term treatment of osteoarthritis.

\section{ACKNOWLEDGEMENTS}

This study was funded by the Rottapharm|Madaus Group. The Authors are grateful to Jean Yves Reginster and Gabriel Herrero-Beaumont, who provided data from the original studies, and to Beatrice Barbetta, who contributed to statistical analysis.

\section{CONFLICT OF INTEREST}

All authors are employees or consultants of the Rottapharm|Madaus Group.

\section{REFERENCES}

[1] Ezzati M, Lopez AD, Rodgers A, Vander HS, Murray CJ. Selected major risk factors and global and regional burden of disease. Lancet 2002; 360: 1347-60.

[2] Rodgers A, Ezzati M, Vander HS, Lopez AD, Lin RB, Murray CJ. Distribution of major health risks: findings from the global burden of disease study. PLoS Med 2004; 1: e27.

[3] Diabetes mellitus: a major risk factor for cardiovascular disease. A joint editorial statement by the American Diabetes Association; The National Heart, Lung, and Blood Institute; The Juvenile Diabetes Foundation International; The National Institute of Diabetes and Digestive and Kidney Diseases; and The American Heart Association. Circulation 1999; 100: 1132-3.

[4] De Backer G, Ambrosioni E, Borch-Johnsen K, et al. European guidelines on cardiovascular disease prevention in clinical practice.
Third Joint Task Force of European and Other Societies on Cardiovascular Disease Prevention in Clinical Practice. Eur Heart J 2003; 24: 1601-10.

[5] Lohmander LS, Roos EM. Clinical update: treating osteoarthritis. Lancet 2007; 370: 2082-4

[6] Borer JS, Pouleur H, Abadie E, et al. Cardiovascular safety of drugs not intended for cardiovascular use: need for a new conceptual basis for assessment and approval. Eur Heart J 2007; 28: 1904-9.

[7] Mancia G, De Backer G, Dominiczak A, et al. 2007 Guidelines for the management of arterial hypertension: The Task Force for the Management of Arterial Hypertension of the European Society of Hypertension (ESH) and of the European Society of Cardiology (ESC). Eur Heart J 2007; 28: 1462-536.

[8] Dougados M. Recommendations for the registration of drugs used in the treatment of osteoarthritis. Ann Rheum Dis 1996; 55: 552-7.

[9] Setnikar I, Rovati LC. Absorption, distribution, metabolism and excretion of glucosamine sulfate. Arzneimittelforschung 2001; 51: 699-725.

[10] Herrero-Beaumont G, Ivorra JA, Del Carmen TM, et al. Glucosamine sulfate in the treatment of knee osteoarthritis symptoms: a randomized, double-blind, placebo-controlled study using acetaminophen as a side comparator. Arthritis Rheum 2007; 56: 555-67.

[11] Reginster JY, Deroisy R, Rovati LC, et al. Long-term effects of glucosamine sulphate on osteoarthritis progression: a randomised, placebo-controlled clinical trial. Lancet 2001; 357: 251-6.

[12] Pavelka K, Gatterova J, Olejarova M, Machacek S, Giacovelli G, Rovati LC. Glucosamine sulfate use and delay of progression of knee osteoarthritis: a 3-year, randomized, placebo-controlled, double-blind study. Arch Intern Med 2002; 162: 2113-23.

[13] Mueller-Fassbender H, Bach GL, Haase W, Rovati LC, Setnikar I. Glucosamine sulfate compared to ibuprofen in osteoarthritis of the knee. Osteoarthritis Cartilage 1994; 2: 61-9.

[14] Qiu GX, Gao SN, Giacovelli G, Rovati L, Setnikar I. Efficacy and safety of glucosamine sulfate versus ibuprofen in patients with knee osteoarthritis. Arzneimittelforschung 1998; 48: 469-74.

[15] Towheed TE, Maxwell L, Anastassiades TP, et al. Glucosamine therapy for treating osteoarthritis. Cochrane Database Syst Rev 2005; (2): CD002946.

[16] Baron AD, Zhu JS, Zhu JH, Weldon H, Maianu L, Garwey WT Glucosamine induces insulin resistance in vivo by affecting GLUT 4 translocation in skeletal muscle. J Clin Invest 1995; 96: 2792801 .

[17] Lassen E. [Glucosamine/cholesterol a possible interaction]. Ugeskr Laeger 2004; 166: 3895.

[18] Altman R, Asch E, Bloch D, et al. Development of criteria for the classification and reporting of osteoarthritis: classification of osteoarthritis of the knee. Diagnostic and Therapeutic Criteria Committee of the American Rheumatism Association. Arthritis Rheum 1986; 29: 1039-49.

[19] Cicero AF, Rovati LC, Setnikar I. Eulipidemic effects of berberine administered alone or in combination with other natural cholesterol-lowering agents. A single-blind clinical investigation. Arzneimittelforschung 2007; 57: 26-30.

[20] Friedewald WT, Levy RI, Fredrickson DS. Estimation of the concentration of low-density lipoprotein cholesterol in plasma, without use of the preparative ultracentrifuge. Clin Chem 1972; 18: 499-502.

[21] National Cholesterol Education Program (NCEP) Expert Panel on Detection, Evaluation, and Treatment of High Blood Cholesterol in Adults (Adult Treatment Panel III). Third Report of the National Cholesterol Education Program (NCEP) Expert Panel on Detection, Evaluation, and Treatment of High Blood Cholesterol in Adults (Adult Treatment Panel III) final report. Circulation 2002; 106: 3143-421.

[22] Grundy SM, Cleeman JI, Merz CN, et al. Implications of recent clinical trials for the national cholesterol education program adult treatment panel III guidelines. Circulation 2004; 110: 227-39.

[23] World Health Organization. Definition and diagnosis of diabetes mellitus and intermediate hyperglicemia: report of a WHO/IDF consultation. Updated 2006, accessed 2011. Available from http://www.who.int/diabetes/publications/en/

[24] Couzin J. Drug safety. FDA panel urges caution on many antiinflammatory drugs. Science 2005; 307: 1183-5. 
[25] Block G, Jensen CD, Norkus EP, et al. Usage patterns, health, and nutritional status of long-term multiple dietary supplement users: a cross-sectional study. Nutr J 2007; 6: 30.

[26] Echard BW, Talpur NA, Funk KA, Bagchi D, Preuss HG. Effects of oral glucosamine and chondroitin sulfate alone and in combination on the metabolism of SHR and SD rats. Mol Cell Biochem 2001; 225: 85-91.

[27] Østergaard LK, Hviid T, Hyllested-Winge JL. [The effect of glucosamine sulphate on the blood levels of cholesterol or triglycerides--a clinical study]. Ugeskr Laeger 2007; 169: 407-10.

[28] Haas MJ, Wong NC, Mooradian AD. Effect of glucosamine on apolipoprotein AI mRNA stabilization and expression in HepG2 cells. Metabolism 2004; 53: 766-71.

[29] Albert SG, Oiknine RF, Parseghian S, Mooradian AD, Haas MJ, McPherson T. The effect of glucosamine on Serum HDL cholesterol and apolipoprotein AI levels in people with diabetes. Diabetes Care 2007; 30: 2800-3.

[30] Shankar RR, Zhu JS, Baron AD. Glucosamine infusion in rats mimics the beta-cell dysfunction of non-insulin-dependent diabetes mellitus. Metabolism 1998; 47: 573-7.

[31] Muniyappa R, Karne RJ, Hall G, et al. Oral glucosamine for 6 weeks at standard doses does not cause or worsen insulin resistance or endothelial dysfunction in lean or obese subjects. Diabetes 2006; 55: 3142-50

[32] Pouwels MJ, Jacobs JR, Span PN, Lutterman JA, Smits P, Tack CJ. Short-term glucosamine infusion does not affect insulin sensitivity in humans. J Clin Endocrinol Metab 2001; 86: 2099-103.

[33] Monauni T, Zenti MG, Cretti A, et al. Effects of glucosamine infusion on insulin secretion and insulin action in humans. Diabetes 2000; 49: 926-35.

[34] Bailey CJ, Turner SL. Glucosamine-induced insulin resistance in L6 muscle cells. Diabetes Obes Metab 2004; 6: 293-8.
[35] Persiani S, Roda E, Rovati LC, Locatelli M, Giacovelli G, Roda A Glucosamine oral bioavailability and plasma pharmacokinetics after increasing doses of crystalline glucosamine sulfate in man. Osteoarthritis Cartilage 2005; 13: 1041-9.

[36] Scroggie DA, Albright A, Harris MD. The effect of glucosaminechondroitin supplementation on glycosylated hemoglobin levels in patients with type 2 diabetes mellitus: a placebo-controlled, doubleblinded, randomized clinical trial. Arch Intern Med 2003; 163: 1587-90.

[37] Tannis AJ, Barban J, Conquer JA. Effect of glucosamine supplementation on fasting and non-fasting plasma glucose an serum insulin concentrations in healthy individuals. Osteoarthritis Cartilage 2004; 12: 506-11.

[38] Anderson JW, Nicolosi RJ, Borzelleca JF. Glucosamine effects in humans: a review of effects on glucose metabolism, side effects, safety considerations and efficacy. Food Chem Toxicol 2005; 43 : 187-201.

[39] Stumpf JL, Lin SW. Effect of glucosamine on glucose control. Ann Pharmacother 2006; 40: 694-8.

[40] Simon RR, Marks V, Leeds AR, Anderson JW. A comprehensive review of oral glucosamine use and effects on glucose metabolism in normal and diabetic individuals. Diabetes Metab Res Rev 2011; 27: 14-27.

[41] Pham T, Cornea A, Blick KE, Jenkins A, Scofield RH. Oral glucosamine in doses used to treat osteoarthritis worsens insulin resistance. Am J Med Sci 2007; 333: 333-9.

[42] Biggee BA, Blinn CM, Nuite M, Silbert JE, McAlindon TE. Effects of oral glucosamine sulphate on serum glucose and insulin during an oral glucose tolerance test of subjects with osteoarthritis. Ann Rheum Dis 2007; 66: 260-2.

(C) Palma dos Reis et al.; Licensee Bentham Open.

This is an open access article licensed under the terms of the Creative Commons Attribution Non-Commercial License (http://creativecommons.org/licenses/by-nc/ 3.0/) which permits unrestricted, non-commercial use, distribution and reproduction in any medium, provided the work is properly cited. 